\title{
Exploring the Ethics of Stature Lengthening as Treatment for Height Dysphoria
}

\author{
Richard C Lee ${ }^{1}$, Mark Aulisio², Raymond W Liu ${ }^{3}$
}

\begin{abstract}
Aim: To promote a discussion on the ethics and justifications of stature lengthening in patients without skeletal deformity. Background: Stature lengthening for height gain in patients without skeletal deformity has stirred controversy within the orthopedic community. However, current literature does not delineate the ethical issues surrounding this procedure. Improvements in the techniques, technology, and safety profile of stature lengthening warrant an ethical discussion to challenge, justify, and guide the use of this surgical procedure.

Review results: Examination of ethical issues leads to the distinction between the dual roles of stature lengthening as a treatment vs an enhancement. The primary focus on stature lengthening as treatment allows for exploration of "height dysphoria" - a psychological burden caused by a dissatisfaction with one's height-as the primary pathology that may justify surgical intervention.

Conclusion: In our opinion, additional work is required to establish "height dysphoria" as a true pathology in order to ethically justify stature lengthening as a legitimate form of treatment. Further discussion is needed to address the ethics of stature lengthening as an enhancement. Clinical significance: This paper addresses salient ethical issues of stature lengthening in patients without skeletal deformity by exploring historical, contemporary, and comparative contexts.

Keywords: Enhancement, Ethics, Height dysphoria, Stature lengthening, Treatment.

Strategies in Trauma and Limb Reconstruction (2020): 10.5005/jp-journals-10080-1502
\end{abstract}

\section{INTRODUCTION}

Surgical limb lengthening, performed by distraction osteogenesis, has been used to address skeletal deformities and limb-length discrepancies caused by congenital abnormalities, trauma, tumor, and infections. ${ }^{1,2}$ However, its potential to cosmetically increase height in an otherwise physically healthy patient has stirred controversy within the orthopedic community. We believe that the key to the controversy is the perception of stature lengthening as either a treatment or an enhancement, as the former implies the presence of pathology while the latter does not. ${ }^{3}$ Providers of stature lengthening attribute such pathology to the psychological burden caused by a dissatisfaction with one's height, coined by orthopedic surgeons and psychologists as "height dysphoria"1,4,5 Despite the controversy, stature lengthening attracts demand around the world. ${ }^{6}$ Advancements in techniques and technologies continue to improve the efficacy and safety of stature lengthening. ${ }^{7}$ The increase in demand and improvement in safety warrant an ethical discussion to challenge, justify, and guide the use of stature lengthening.

There is a dearth in the current literature involving any formal ethical discussions on the role of stature lengthening in today's practice. The available literature on stature lengthening primarily consists of Level IV therapeutic studies that discuss the outcomes of different methods. ${ }^{1,4,8-15}$ This paper aims to discuss the controversy of stature lengthening by addressing salient ethical points while providing historical and comparative contexts. To do this effectively, the paper limits its focus to addressing stature lengthening as a treatment to adequately capture the justifications for its current practice. By establishing the ethical groundwork for stature lengthening as a legitimate treatment in patients without skeletal deformity, a further debate may ensue to discuss its role as an enhancement in future discussions.
${ }^{1-3}$ Department of Bioethics, Case Western Reserve University School of Medicine, Cleveland, Ohio, United States

${ }^{3}$ Division of Pediatric Orthopaedics, University Hospitals Rainbow Babies and Children's Hospital, Cleveland, Ohio, United States

Corresponding Author: Richard C Lee, Case Western Reserve University School of Medicine, Cleveland, Ohio, United States, Phone: +1 216368 2000, e-mail: richard.c.lee2@case.edu

How to cite this article: Lee RC, Aulisio M, Liu RW. Exploring the Ethics of Stature Lengthening as Treatment for Height Dysphoria. Strategies Trauma Limb Reconstr 2020;15(3):163-168.

Source of support: Nil

Conflict of interest: None

\section{Current State of Stature Lengthening Historical Background}

Although stature lengthening is a relatively recent procedure, the general practice of limb lengthening dates back over a century. In 1905, Codivilla first wrote about limb lengthening for deformity correction and its effects on surrounding soft tissues. ${ }^{16}$ In 1921, Putti introduced the technique of gradual, continuous traction for femoral lengthening. ${ }^{17}$ The next milestone in limb lengthening advancements occurred in the 1950s-60s through the pioneering works of Ilizarov with the application of circular external fixators and the development of the principles of distraction osteogenesis, which became the prime vehicle for limb lengthening after news of his technique disseminated in the early 1980s. ${ }^{7}$ In the 1970s-80s, Wagner introduced a telescopic monolateral external fixator and 
briefly popularised the technique of rapid distraction and large soft tissue release in the Western world. ${ }^{18,19}$ The turn of the century witnessed a paradigm shift toward implantable nails designed with controlled lengthening mechanisms. These new devices include the Bliskunov telescopic nail (1983), Fitbone (1991), Albizzia (1994), Intramedullary Skeletal Kinetic Distractor (2001), Phenix (2000s), and the PRECICE nail (2011). ${ }^{9}$ Although the earlier nails experienced issues with pain and rate control, the newer nails have undergone iterations of improvements, and as the technology of limb lengthening continues to advance, the feasibility, safety profile, and popularity of stature lengthening follow. ${ }^{20}$ The shift toward implantable nails showed improvements in overall satisfaction, pain reduction, physical therapy, and cosmetic appearance. ${ }^{21}$

\section{Patient Profile}

The mean age of patients undergoing stature lengthening is in the mid-20s, with a range between teenage and $40+$ years (Table 1). Patients also tend to be males, but the proportion is not exact based on limited therapeutic studies. Although the majority of patients undergoing stature lengthening are perceived to have short stature (e.g., below the 5th percentile for age and gender), ${ }^{1}$ height in its absolute value is not the main indication for this procedure. Rather, these patients share the underlying condition of "height dysphoria", in which the person's height severely affects his or her psychological well-being. ${ }^{5}$ Providers have emphasised the importance that patients undergo a psychological evaluation to confirm the presence of height dysphoria and exclude more severe psychiatric conditions. ${ }^{6,8}$ Additional exclusion criteria surround physical conditions to mitigate complication risks. These exclusions include heavy smoking history, presence of open physis, and metabolic diseases. ${ }^{12}$

\section{Risks and Complications}

Risks and complications include those inherent to lengthening and those related to the method of distraction. General complications including soft tissue contracture, neurovascular injury, joint subluxation or dislocation, poor regenerate bone, and premature consolidation. Complications surrounding external fixators include pin site infections, associated deep tissue infections, pain and contracture from soft tissue tethering of the pins, and collapse of regenerate bone after frame removal. ${ }^{4,9}$ Studies that utilised the llizarov external device found complication rates ranging from 37 to $70 \%$, coupled with reoperation rates at 28 to $35 \%$. 8,13
Complications specific to intramedullary methods involve failures in the function and integrity of the implantable nail used. The Albizzia nail is notable for the pain associated with its ratchet mechanism requiring rotation of the limb to achieve lengthening of the nail. ${ }^{9}$ The Intramedullary Skeletal Kinetic Distractor required less rotational movement to achieve lengthening, but it has been shown to have risks of unpredictable "runaway" lengthening rates, reported as high as $5 \mathrm{~mm} /$ day despite a goal of $\leq 1 \mathrm{~mm} /$ day, causing pain and stress on the surrounding soft tissue. ${ }^{22}$ The Fitbone and PRECICE nails avoid the rotational driving mechanism by utilising implanted and magnetic lengthening mechanisms, respectively. Failure to distract and breakage of the nail have been seen in all implantable nails, including the Fitbone and PRECICE series. ${ }^{22}$ However, the complication rates generally fall below those seen in external fixation methods in comparison studies, with rates ranging from 15.4 to $28 \%$ depending on the type of implant used. ${ }^{23-25}$

Compared to patients treated for achondroplasia, patients without skeletal deformities experience higher complication rates while undergoing stature lengthening. ${ }^{14,20}$ This is likely related to the improved soft tissue compliance in achondroplasia, where the soft tissues are thought to have developed in anticipation of a longer bone..$^{20}$ Thus, it is important to adhere to strict selection criteria for otherwise healthy persons undergoing stature lengthening. However, as the overall complication rates improve with advanced intramedullary technology, the prospect of patients pursuing stature lengthening is likely to grow.

\section{Outcomes}

Table 1 summarises the gain of height in both mean and range values specific to patients undergoing stature lengthening without any skeletal deformities and discrepancies. The quantity of height gained through stature lengthening depends on the involvement of tibiae, femora, or both. The majority of available published therapeutic studies involve tibiae lengthening with the use of external fixators. Given that external fixation has technical advantages in the tibia while intramedullary nailing has advantages in the femur, more publications in femoral lengthening are expected in the coming years.

Qualitative outcome measures based on height gain and the presence/absence of complications found that most patients obtain excellent results, although some have been noted to have only good or satisfactory results. ${ }^{8,13}$ In the backdrop of risks and complications associated with stature lengthening, patients report

Table 1: Demographics and results of stature lengthening in patients without skeletal deformities

\begin{tabular}{lllll}
\hline Studies & Mean age & Gender & Mean increase $(\mathrm{cm})$ & Increase range $(\mathrm{cm})$ \\
\hline Guerreschi and Tsibidakis $^{1}$ & 24.8 & $36 \mathrm{M}, 27 \mathrm{~F}$ & $5-11$ & $5-11$ \\
Catagni et al. $^{4}$ & 25.8 & $32 \mathrm{M}, 22 \mathrm{~F}$ & 7 & $2-13$ \\
Novikov et al. $^{8}$ & 25 & $65 \mathrm{M}, 66 \mathrm{~F}$ & 6.9 & $2.7-6.5$ \\
Paley et al. $^{9}$ & 29.7 & $11 \mathrm{M}, 4 \mathrm{~F}$ & 4.63 & $10-13$ \\
Aldegheri and Dall'Oca $^{10}$ & 17.6 & $3 \mathrm{M}, 2 \mathrm{~F}$ & 11.5 & $3.5-12$ \\
Emara et al. $^{15}$ & 27 & $25 \mathrm{M}, 6 \mathrm{~F}$ & 7.6 & $\mathrm{~N} / \mathrm{A}$ \\
Kocaoglu et al. $^{12}$ & 30 & $24 \mathrm{M}, 8 \mathrm{~F}$ & 7.5 & $4-11$ \\
Elbatrawy and Ragab $^{13}$ & 26 & $36 \mathrm{M}, 16 \mathrm{~F}$ & 6.9 & $\mathrm{~N} / \mathrm{A}$ \\
Koczewski et al. $^{14}$ & 18 & $\mathrm{~N} / \mathrm{A}$ & 14.8 &
\end{tabular}

M, male; F, female 
high rates of satisfaction with the result along with improvements in self-esteem and self-confidence..$^{1,8,10,11,13}$ They would also undergo the procedure again and recommend it to others. ${ }^{8}$

In a study that evaluated patients who had undergone both external and intramedullary methods of limb lengthening, the patients showed a unanimous preference for intramedullary devices with respect to overall satisfaction, reduced pain, ease of physical therapy, and better cosmetic appearance. ${ }^{21}$ This is consistent with the lower rates of mild complications seen in intramedullary nails.

\section{Ethical Considerations}

The bioethicist Aquino writes, "Socio-cultural and scientific developments can change the societal as well as the moral value of a certain practice." ${ }^{26}$ Although stature lengthening in patients without skeletal deformity has been a controversial topic in the past decades, the new advancements in intramedullary nail technology and the growing popularity of the procedure warrant a reconsideration of the moral question behind stature lengthening. The technical papers that describe the methods and outcomes of stature lengthening mention the existence of controversy behind the practice but fall short of delineating what exactly strikes our moral chord. This section helps to highlight the salient ethical concerns that surround stature lengthening to guide discussion on the future direction of the practice.

\section{Treatment Medicalisation}

The view of stature lengthening as treatment implies the presence of pathology that warrants correction. Part of this implication stems from the medicalisation of height and its associated psychological effects. Treatment medicalisation involves the adoption of medical language and frameworks to describe and understand a problem so that it may be treated with medical and surgical interventions. ${ }^{27}$ Such an example of treatment medicalisation can be seen in the use of standard deviations to provide a framework for understanding height distribution within a population. Surgeons viewing height as a medical concept may then refer to ideal measurements and proper ratios in an effort to "objectively" approach a perceived problem. ${ }^{26}$ This results in height values that are labeled as "normal" vs "abnormal" (e.g., below the 5th percentile). The label of "abnormal" then lends itself to further medical jargon, resulting in terms, such as "constitutional short stature" and "height dysphoria" to label patients who are screened for stature lengthening.

While the adoption of medical terms attempts to provide consistency and objectivity in the discussion of perceived conditions, it does not guarantee validity. Critics of stature lengthening have questioned the legitimacy of medicalising height in the absence of other skeletal abnormalities. ${ }^{2}$ The standardisation of height as an objective approach cannot escape the subjective factors rooted in cultural and ethnic differences. For example, what is labeled as "normal" distribution in height in Northern European populations certainly does not apply to other populations. ${ }^{28}$

Moreover, criticism against stature lengthening challenges the validity of height dysphoria and the impact of surgical interventions on this psychological condition. Although the practice of screening surgical candidates with psychologists has become nearly universal among published therapeutic studies, critics note that the details on the definition, measurement, and evaluation of height dysphoria are poorly documented. ${ }^{2}$ Thus, the criticism does not take issue with the overall treatment medicalisation of stature lengthening but, rather, with the degree to which current documentation fails to describe and justify the most important indication for this procedure. In our opinion, if height dysphoria is to become a major indication for defending stature lengthening, then further work is needed to clarify not only its parameters, but how surgery can impact it with objective outcomes. If stature lengthening is to be justified with treatment medicalisation, we believe it must be done appropriately to address the standards of the scientific community.

\section{Psychosocial Impact of Height}

Extensive research already exists on the psychosocial impact of height. Studies show both a positive and a negative correlation between height and social outcomes. Height is positively linked to higher income, education, and marriage potential..$^{29,30}$ On the other hand, shorter height has been found to be associated with higher rates of depression and suicide risk, especially in younger men. ${ }^{31,32}$ Virtual reality simulations altering height perspectives have correlated reductions in height with increases in paranoia and vulnerability. ${ }^{33}$ If short stature is viewed as a true deviation, then it may be viewed as a disability that impacts the patient's perception of the self and surroundings. ${ }^{10}$ Interestingly, the psychosocial effects of height are more related to relative height comparisons rather than to absolute height values. ${ }^{34}$ This supports how height dysphoria may be independent of absolute height, thus supporting height dysphoria-not short stature-as the main indication for stature lengthening. If height dysphoria is shown to be an objective condition that can be impacted by stature lengthening, then it may be viewed as a pathology that can be justifiably corrected by surgical intervention.

\section{Height Dysphoria vs Body Dysmorphic Disorder}

Current orthopedic literature attempts to distinguish height dysphoria from body dysmorphic disorder (BDD), going as far as labeling BDD as an absolute contraindication for stature lengthening., ${ }^{1,5} 8$ BDD is defined by the Diagnostic and Statistical Manual of Mental Disorders, 5th Edition as the presence of the following criteria: ${ }^{35}$

1. Preoccupation with one or more perceived defects or flaws in physical appearance that are not observable or appear slight to others.

2. At some point during the course of the disorder, the individual has performed repetitive behaviors (e.g., mirror checking, excessive grooming, skin picking, reassurance seeking) or mental acts (e.g., comparing his or her appearance with that of others) in response to the appearance concerns.

3. The preoccupation causes clinically significant distress or impairment in social, occupational, or other important areas of functioning.

4. The appearance preoccupation is not better explained by concerns with body fat or weight in an individual whose symptoms meet diagnostic criteria for an eating disorder.

On examination, the criteria for BDD significantly overlap with those of height dysphoria. If height dysphoria is truly independent of absolute height, then the perceived "flaw" in height may not be observable to others (Criterion 1). The psychological burden of height dysphoria may result in daily preoccupation and significant distress due to one's height (Criteria 2 and 3). Given the similarities and the lack of a clear definition for height dysphoria, it may be premature to exclude all BDD patients from stature lengthening. The current exclusion of BDD is based on the assumption that BDD symptoms worsen after cosmetic procedures due to an 
indefinite dissatisfaction with multiple surgical outcomes or a shift in preoccupation with a different body part. ${ }^{36}$ However, recent studies in the cosmetic field stratify BDD by mild, moderate, and severe tiers based on validated screening tools and show that patients with mild-to-moderate BDD with realistic expectations have good outcomes from cosmetic procedures while patients with severe BDD continue to have indefinite dissatisfaction. ${ }^{37-39}$ Thus, stature lengthening indications may require the inclusion of mild-to-moderate BDD patients with careful consideration of predicted satisfaction and patient safety, rather than a blanket exclusion of BDD. ${ }^{40}$ Alternatively, providers of stature lengthening and associated psychologists may further redefine height dysphoria to clearly delineate its features from those of mild-to-moderate BDD. Regardless, the ill-defined position of height dysphoria in the contextual spectrum of BDD does not diminish the role of stature lengthening as an effective surgical treatment for the psychological burden attributed to dissatisfaction with one's own height.

\section{Achondroplasia as Parallel Comparison}

Using stature lengthening to treat height dysphoria as a true pathology can be supported by understanding how the practice of limb lengthening is acceptable in treating patients with skeletal deformities, such as achondroplasia. Several parallels can be drawn between patients with achondroplasia who undergo limb lengthening and patients with height dysphoria. First, limb lengthening in achondroplasia is an elective procedure that is based on personal preferences and well-informed decisionmaking. ${ }^{41}$ Second, the increase in performing limb lengthening in achondroplasia came only after techniques became safer and more reliable. ${ }^{42,43}$ This alludes to the possibility that the controversy in limb lengthening in achondroplasia rested less on the action of lengthening itself as it did on the risks and complications that put patients at harm. Thus, reducing complications and improving technical approaches made surgical intervention more acceptable.

The case of achondroplasia, however, differs from the case of height dysphoria in that limb lengthening addressed not only height gain but also improvements in physiological proportions, joint and spine alignment, and overall physical function. ${ }^{44-46}$ Patients with achondroplasia also experienced fewer soft tissue complications due to more ligament and muscle laxity compared to other short stature patients. ${ }^{47}$ Still, limb lengthening in patients with achondroplasia resulted in improved psychological and emotional state, including improved quality of life and self-esteem. ${ }^{20,43,48,49}$

In correcting height in patients with achondroplasia, surgical intervention addressed both the physical and psychological state. The pathology in achondroplasia is more apparent and thus harbors fewer questions about whether limb lengthening is a form of treatment. In stature lengthening, however, the legitimacy of height dysphoria as pathology is implied but not yet concrete. Exploration of height dysphoria should go deeper than a brief mention in the small therapeutic studies that are susceptible to selection and assessment biases. ${ }^{2}$ However, it is evident that groundwork for this pathology already exists. Studies regarding the association between height and psychosocial outcomes suggest that height can be a modifiable risk factor if done through safe, reliable methods of surgical correction. As techniques of limb lengthening improved the safety and outcomes in patients with achondroplasia, the perception and judgment of the procedure itself slowly changed to one of understanding and respect for personal choice. ${ }^{41}$ Perhaps this is the path that stature lengthening in patients without skeletal deformity will also take as new advancements in intramedullary technology and safer techniques are now helping to bring light to the reasons why controversy around the practice exists.

\section{Ethical Analysis}

As noted above, the condition of height dysphoria needs to be better characterised in order for a careful risk-benefit assessment of stature lengthening to be done. Assuming that height dysphoria can be reasonably well described and that limb lengthening itself can be supported as a possible treatment option that should be offered to certain patients, further ethical analysis would be required to inform its use in particular cases. This analysis centers on considerations of patient autonomy, beneficence, and nonmaleficence. $^{50}$

Patients with height dysphoria should be free to make an informed decision to undergo stature lengthening. Decisionmaking capacity must include the patient's ability to communicate, understand, appreciate, and rationalize the indications, risks, and benefits of the treatment in question..$^{51}$ This entails a proper understanding of the duration of the treatment and possible complications, but more importantly, ensuring that the patient has decision-making capacity free of any coercion or undue influence. The mere presence of a psychological condition fixated on height does not automatically undermine one's capacity to make a choice to pursue stature lengthening. Such a patient should undergo a thorough psychological evaluation to ensure that the patient is of good insight and rationality. On the other hand, a patient with severe BDD would not be able to appropriately consent to stature lengthening since the patient's warped perspective of an imagined bodily defect and lack of insight on the goals of surgery disqualify the patient from properly understanding, appreciating, and rationalising the need for surgery. ${ }^{38}$ This underscores the importance of having a low threshold for preoperative psychological evaluation of any patient considering stature lengthening. Such threshold will help to ensure that patients whose height dysphoria may undermine decision capacity altogether will be prevented from being allowed to make a choice they lack the capacity to make.

Considerations of beneficence and non-maleficence go hand-in-hand in the case of stature lengthening. Based on the risks and complications behind the procedure, proceeding with surgery requires careful consideration of the risk-benefit ratio. As discussed, patients undergoing stature lengthening experience improvements in self-esteem in conjunction with height gain. In addition, treatment of height dysphoria may benefit the patient through decreasing depression and suicide rates while improving socioeconomic gains. ${ }^{29-32}$ However, the associated complications of infections, nonunion, deformities, and soft tissue contractures appear to challenge the limits of non-maleficence. Aldegheri et al. state that the benefits gained through stature lengthening outweigh the painful costs that the patients experience. ${ }^{10}$ The high patient satisfaction ratings with the procedure despite risks and complications reinforce this claim. The safer practices of intramedullary nails and improved parameters behind lengthening techniques continue to tip the risk-benefit ratio in favor of beneficence. The prevention of harm (non-maleficence) can be further reinforced with guidelines to provide committed care for the patients throughout the entire course of the treatment, from adequate screening to proper rehabilitation management. ${ }^{6}$

Despite the increasing safety profile and high satisfaction rates with using intramedullary nails in stature lengthening, non-surgical interventions should also be investigated to treat height dysphoria. 
The principle of non-maleficence warrants initial attempts with less-invasive interventions prior to resorting to surgery. Possible avenues for addressing height dysphoria may reside in psychotherapy or pharmacotherapy. However, current literature in stature lengthening has no mention of prior psychotherapy, pharmacotherapy, or other non-surgical alternatives. For psychotherapy, cognitive behavior therapy (CBT) may be effective in changing the patient's perception of his or her height. For pharmacotherapy, although the use of recombinant human growth hormone therapy is not possible in adults as the closure of bone physes prevents this option, ${ }^{52}$ selective serotonin reuptake inhibitors (SSRIs) may help manage the psychological distresses of height dysphoria. Both CBT and SSRIs have already shown varying degrees of efficacy in patients with BDD, but they remain untested and undocumented in patients with height dysphoria. ${ }^{53}$ Given the potential of psychotherapy and pharmacotherapy, it is our opinion that further research is required to provide evidence for these potential alternatives. Although surgical stature lengthening is effective in physical and psychological outcomes, we believe its success alone should not bar the pursuit of non-invasive alternatives if, and only if, these non-invasive alternatives are proven to be safe and effective.

Beneficence and non-maleficence in the form of the risk-benefit ratio should be addressed by both the patient and the provider. The patient should be able to delineate the risks and benefits of stature lengthening as part of decision-making capacity. However, sole reliance on the patient to perform this calculus should be avoided as the high satisfaction rates despite high complication rates suggest patient bias in favor of surgery. ${ }^{1,8,10,11,13}$ Rather, orthopedic providers should play a significant role in determining a clear risk-benefit ratio for each patient based on individual projected outcomes and anticipated complications. The orthopedic provider, in consultation with a psychologist, should be the gatekeeper to the procedure as patient values may be predetermined in favor of surgery. Once both the patient and provider are in agreement, the realistic expectations that stem from the therapeutic alliance can prevent unnecessary surgeries.

\section{ConcLusion}

Could stature lengthening be ethically justified if the patient does not have height dysphoria? As the focus of this paper viewed stature lengthening through the perspective of treatment, it did not address how the debate would change if the procedure were discussed as an enhancement, in which the need for pathology no longer becomes required. Such discussion requires evaluation of the role and limits of surgeons in drawing their own line of social and medical responsibilities. This paper also did not explore the societal repercussions of expanding the incidence of stature lengthening, including cost coverage and changes in social norms. These are all issues that warrant further discussion within the ethics and orthopedic communities. However, clarification and acceptance of stature lengthening as treatment must be made first before delving into the more controversial topic of stature lengthening as an enhancement.

Technical and technological advancements in the area of intramedullary approaches have greatly improved the risks and complications surrounding stature lengthening. When viewing this advancement in parallel to how limb lengthening became more widespread in treating patients with achondroplasia, it is a matter of time before concerns about the non-maleficence in stature lengthening subside in relation to the beneficence it provides. Additional research is required to look into the outcomes of intramedullary nails specifically in patients with height dysphoria without any other skeletal deformities, as most of the current literature involves external fixators. However, focus should also be given to advancing the literature regarding height dysphoria in terms of its diagnosis, difference from BDD, and alternative treatment approaches. By further defining, measuring, and evaluating height dysphoria in relation to both the patient and the procedure, the ethical justifications for stature lengthening can be facilitated by establishing the procedure's role as treatment for a true pathology.

\section{References}

1. Guerreschi F, Tsibidakis $H$. Cosmetic lengthening: what are the limits? J Child Orthop 2016;10(6):597-604. DOI:10.1007/s11832-0160791-z.

2. Leopold SS, Sabharwal S. Editorial: indications - on the fringes and in the mainstream. Clin Orthop Relat Res 2014;472(11):3247-3249. DOI: 10.1007/s11999-014-3927-9.

3. Bostrom N, Roache R. Human enhancement : ethical issues in human enhancement. In: Ryberg J, Petersen TS, Wolf C, eds. New Waves in Applied Ethics. London, UK: Palgrave-Macmillan; 2007.

4. Catagni MA, Lovisetti L, Guerreschi F, et al. Cosmetic bilateral leg lengthening: experience of 54 cases. J Bone Joint Surg $\mathrm{Br}$ 2005;87(10):1402-1405. DOI: 10.1302/0301-620X.87B10.16468.

5. Vishwanathan K, Nimbalkar S. Cosmetic limb lengthening in a patient of normal stature: ethical considerations. Indian J Med Ethics 2017;2(1):45-48. DOI: 10.20529/IJME.2017.009.

6. Patel M. Cosmetic limb lengthening surgery: the elephant in the room. Harm minimization not prohibition. J Limb Lengthen Reconstr 2017;3:73-74. https://www.jlimblengthrecon.org/text. asp?2017/3/2/73/213565.

7. Paley D. The llizarov technology revolution: history of the discovery, dissemination, and technology transfer of the llizarov method. J Limb Lengthen Reconstr 2018;4(2):115-128. DOI: https://www. jlimblengthrecon.org/text.asp?2018/4/2/115/253395.

8. Novikov KI, Subramanyam KN, Muradisinov SO, et al. Cosmetic lower limb lengthening by Ilizarov apparatus: what are the risks? Clin Orthop 2014;472(11):3549-3556. DOI: 10.1007/s11999-014-3782-8.

9. Paley D, Harris M, Debiparshad K, et al. Limb lengthening by implantable limb lengthening devices. Tech Orthop 2014;29(2):72-85. DOI: $10.1097 /$ BTO.0000000000000072.

10. Aldegheri R, Dall'Oca C. Limb lengthening in short stature patients. J Pediatr Orthop B 2001;10(3):238-247. DOI: 10.1097/00009957200107000-00015.

11. Emara K, Al Kersh MA, Emara AK. Long term self esteem assessment after height increase by lengthening and then nailing. Acta Orthop Belg 2017;83(1):40-44.

12. Kocaoglu M, Bilen FE, Eralp IL, et al. Results of cosmetic lower limb lengthening by the lengthening over nail technique. Acta Orthop Belg 2017;83(2):231-244.

13. Elbatrawy $Y$, Ragab IMA. Safe cosmetic leg lengthening for short stature: long-term outcomes. Orthopedics 2015;38(7):e552-560. DOI: 10.3928/01477447-20150701-51.

14. Koczewski P, Shadi M, Napiontek M. The surgical treatment of short stature: management strategy and local experience. Ortop Traumatol Rehabil 2002;4(4):421-426.

15. Emara K, Farouk A, Diab R. Ilizarov Technique of Lengthening and Then Nailing for Height Increase. J Orthop Surg 2011;19(2):204-208. DOI: $10.1177 / 230949901101900215$.

16. Codivilla A. On the means of lengthening, in the lower limbs, the muscles and tissues which are shortened through deformity. JBJS 1905;s2-2(4):353-369.

17. Putti V. The operative lengthening of the femur. 1921. Clin Orthop Relat Res. 1990;(250):4-7.

18. Birch JG. A brief history of limb lengthening. J Pediatr Orthop 2017;37 Suppl 2:S1-S8. DOI: 10.1097/BPO.0000000000001021. 
19. Hasler CC, Krieg AH. Current concepts of leg lengthening. J Child Orthop 2012;6(2):89-104. DOI: 10.1007/s11832-012-0391-5.

20. Kim S-J, Pierce W, Sabharwal S. The etiology of short stature affects the clinical outcome of lower limb lengthening using external fixation. A systematic review of 18 trials involving 547 patients. Acta Orthop 2014;85(2):181-186. DOI: 10.3109/17453674.2014.899856.

21. Landge V, Shabtai L, Gesheff M, et al. Patient satisfaction after limb lengthening with internal and external devices. J Surg Orthop Adv 2015;24(3):174-179.

22. Paley D. PRECICE intramedullary limb lengthening system. Expert Rev Med Devices 2015;12(3):231-249. DOI: 10.1586/17434440.2015. 1005604.

23. Laubscher M, Mitchell C, Timms A, et al. Outcomes following femoral lengthening: An initial comparison of the Precice intramedullary lengthening nail and the LRS external fixator monorail system. Bone Joint J 2016;98-B(10):1382-1388. DOI: 10.1302/0301-620X.98B10. 36643.

24. Wagner P, Burghardt RD, Green SA, et al.PRECICE ${ }^{\circledR}$ magnetically-driven, telescopic, intramedullary lengthening nail: pre-clinical testing and first 30 patients. SICOT J 2017;3:19. DOI: 10.1051/sicotj/2016048.

25. Accadbled F, Pailhé R, Cavaignac $E$, et al. Bone lengthening using the Fitbone $\left({ }^{\oplus}\right)$ motorized intramedullary nail: the first experience in France. Orthop Traumatol Surg Res 2016;102(2):217-222. DOI: 10.1016/j.otsr.2015.10.011.

26. Aquino YSJ. "Big eye" surgery: the ethics of medicalizing Asian features. Theor Med Bioeth. 2017;38(3):213-225. DOI: 10.1007/s11017017-9395-y.

27. Conrad P. Medicalization and Social Control. Annu Rev Sociol 1992;18(1):209-232. DOI: 10.1146/annurev.so.18.080192.001233.

28. Roser M, Appel C, Ritchie H. Human Height. Our World Data. October 2013. https://ourworldindata.org/human-height. Accessed November 11, 2019.

29. Deaton A, Arora R. Life at the top: the benefits of height. Econ Hum Biol 2009;7(2):133-136. DOI: 10.1016/j.ehb.2009.06.001.

30. Smits J, Monden CWS. Taller Indian women are more successful at the marriage market. Am J Hum Biol 2012;24(4):473-478. DOI: 10.1002/ ajhb.22248.

31. Magnusson PKE, Gunnell D, Tynelius P, et al. Strong inverse association between height and suicide in a large cohort of swedish men: evidence of early life origins of suicidal behavior? Am J Psychiatry 2005;162(7):1373-1375. DOI: 10.1176/appi.ajp.162.7.1373.

32. Krupnik V, Cherkasova MV. Size matters stature is related to diagnoses of depression in young military men. SAGE Open 2014;4(3):2158244014542783. DOI: 10.1177/2158244014542783.

33. Freeman D, Evans N, Lister R, et al. Height, social comparison, and paranoia: an immersive virtual reality experimental study. Psychiatry Res 2014;218(3):348-352. DOI: 10.1016/j.psychres.2013.12.014.

34. Carrieri V, De Paola M. Height and subjective well-being in Italy. Econ Hum Biol 2012;10(3):289-298. DOI: 10.1016/j.ehb.2011.12.002.

35. Obsessive-Compulsive and Related Disorders. In: Diagnostic and Statistical Manual of Mental Disorders. DSM Library. American Psychiatric Association; 2013. DOI: 10.1176/appi. books.9780890425596.dsm06.
36. Kuhn H, Cunha PR, Matthews NH, et al. Body dysmorphic disorder in the cosmetic practice. G Ital Dermatol Venereol 2018;153(4):506-515. DOI: 10.23736/S0392-0488.18.05972-2.

37. Panayi A. The prevalence of body dysmorphic disorder in patients undergoing cosmetic surgery: a systematic review. Psychiatr Danub 2015;27 Suppl 1:S438-444.

38. Higgins $S$, Wysong A. Cosmetic surgery and body dysmorphic disorder - an update. Int J Womens Dermatol 2018;4(1):43-48. DOI: 10.1016/j.ijwd.2017.09.007.

39. Bowyer L, Krebs G, Mataix-Cols D, et al. A critical review of cosmetic treatment outcomes in body dysmorphic disorder. Body Image 2016;19:1-8. DOI: 10.1016/j.bodyim.2016.07.001.

40. Tadisina KK, Chopra K, Singh DP. Body dysmorphic disorder in plastic surgery. eplasty 2013;13. https://www.ncbi.nlm.nih.gov/pmc/articles/ PMC3693597/. Accessed October 3, 2019.

41. Gross R. Limb lengthening for stature: another view. J Pediatr Orthop 2005;25(1):128-129. DOI: 10.1097/00004694-200501000-00027.

42. Leg lengthening in achondroplasia. Lancet 1988;1(8593):1032.

43. Lavini F, Renzi-Brivio L, de Bastiani G. Psychologic, vascular, and physiologic aspects of lower limb lengthening in achondroplastics. Clin Orthop Relat Res 1990;(250):138-142.

44. Schiedel F, Rödl R. Lower limb lengthening in patients with disproportionate short stature with achondroplasia: a systematic review of the last 20 years. Disabil Rehabil 2012;34(12):982-987. DOI: 10.3109/09638288.2011.631677.

45. Stanitski C. Limb Lengthening for Stature. J Pediatr Orthop 2004;24(5):593-594.

46. Park HW, Kim HS, Hahn SB, et al. Correction of lumbosacral hyperlordosis in achondroplasia. Clin Orthop Relat Res 2003;414:242249. DOI: 10.1097/01.blo.0000081936.75404.a4.

47. Park K-W, Garcia RN, Rejuso CA, et al. Limb lengthening in patients with achondroplasia. Yonsei Med J 2015;56(6):1656-1662. DOI: 10.3349/ymj.2015.56.6.1656.

48. Aldegheri R, Trivella G, Renzi-Brivio $L$, et al. Lengthening of the lower limbs in achondroplastic patients. A comparative study of four techniques. J Bone Joint Surg Br 1988;70(1):69-73. DOI: 10.1302/0301620X.70B1.3339063.

49. Donaldson J, Aftab S, Bradish C. Achondroplasia and limb lengthening: results in a UK cohort and review of the literature. J Orthop 2015;12(1):31-34. DOI: 10.1016/j.jor.2015.01.001.

50. Beauchamp TL, Childress JF. Principles of Biomedical Ethics. Oxford University Press; 2009.

51. Dastidar JG, Odden A. How do I determine if my patient has decisionmaking capacity? https://www.the-hospitalist.org/hospitalist/ article/124731/how-do-i-determine-if-my-patient-has-decisionmaking-capacity. Accessed July 1, 2019.

52. Food and drug administration. FDA approves humatrope for short stature. Fed Regist 2003;68:24003. https://www.accessdata.fda.gov/ drugsatfda_docs/appletter/2003/19640se1-033ltr.pdf.

53. Phillipou A, Rossell SL, Wilding HE, et al. Randomised controlled trials of psychological \& pharmacological treatments for body dysmorphic disorder: a systematic review. Psychiatry Res 2016;245:179-185. DOI:10.1016/j.psychres.2016.05.062. 